\section{Isopolar phosphonate analogue of adenosine diphosphate ribose.}

\section{Kim Van derpoorten and Marie E Migaud}

\section{Supporting Information}

\section{Experimental section}

HPLC runs were performed on a Gilson Anachem system with UV detector operating at $254 \mathrm{~nm}$. The HPLC chromatography columns, purchased from Supelco. Anion exchange chromatography was run on a SAX (200 x 4.5 $\mathrm{mm}, 5 \mu$ ) column with an isocratic elution using phosphate buffer $\left(\mathrm{KH}_{2} \mathrm{PO}_{4} 50 \mathrm{mM}, \mathrm{pH}=3.5,5 \% \mathrm{MeOH}\right)$ at a flow rate of $1 \mathrm{~mL} / \mathrm{min}$ while reversed-phase chromatography was run on a $\mathrm{RP}_{-} \mathrm{C}_{18}$ column. For method 1, elutions were achieved by increasing the methanol content of a formic acid solution $(50 \mathrm{mM}, \mathrm{pH}=6.0)$ from $5 \%$ to $30 \%$ in a linear gradient over $30 \mathrm{~min}$. For method 2, a solution of formic acid $(50 \mathrm{mM}, \mathrm{pH}=6.0)$ containing $5 \%$ of methanol was used isocratically. The ${ }^{1} \mathrm{H},{ }^{13} \mathrm{C}$ and ${ }^{31} \mathrm{P}$ NMR spectra were recorded in $\mathrm{CDCl}_{3}$ or in $\mathrm{D}_{2} \mathrm{O}$ on a Brucker AC $300 \mathrm{MHz}$ spectrometer. HMQC and ${ }^{1} \mathrm{H}-{ }^{1} \mathrm{H}$ COSY experiments were performed on a Brucker AMX 500 spectrometer. TMS (0 $\left.\mathrm{ppm},{ }^{1} \mathrm{H} \mathrm{NMR}\right), \mathrm{CDCl}_{3}$ (77 ppm, $\left.{ }^{13} \mathrm{C} \mathrm{NMR}\right)$ and $(\mathrm{EtO})_{3} \mathrm{PO}$ (0 ppm, ${ }^{31} \mathrm{P}$ NMR) were used as internal references. The chemical shifts are reported in $\delta$ p.p.m. $J$ values are recorded in $\mathrm{Hz}$. Liquid secondary ion mass spectra (LSIMS) were recorded in a 3-nitrobenzylalcohol matrix with an effective accelerating voltage of $17 \mathrm{kV}$ on a VG Autospec (Micromass, UK) instrument. Electrospray mass spectra (ES-MS) was performed on a VG Quattro Triple Quadrupole Mass Spectrometer with a flow rate of 20 $\mu \mathrm{L} / \mathrm{min}$ and a capillary voltage of $3.5 \mathrm{kV}$. Reactions were performed under dry argon atmosphere.

Allyloxy diisopropylamino trimethylsilylethynylphosphine (3)

Trimethylsilylacetylene $(0.34 \mathrm{~g}, 3.45 \mathrm{mmol})$ dissolved in THF $(40 \mathrm{~mL})$ was treated with $n$-butyllithium $(2 \mathrm{M}$ in hexane, $1.9 \mathrm{~mL}, 3.80 \mathrm{mmol})$ at $-78^{\circ} \mathrm{C}$. The formed trimethylsilylacetylide anion was added dropwise via cannula to a solution of allyloxychlorodiisopropylaminophosphine in THF $(40 \mathrm{~mL})$ cooled at $-78^{\circ} \mathrm{C}$. The resulting mixture was stirred at $-78^{\circ} \mathrm{C}$ for $2 \mathrm{~h}$, diluted with $\mathrm{CH}_{2} \mathrm{Cl}_{2}(40 \mathrm{~mL})$ and washed with $\mathrm{NaHCO}_{3}(5 \%)$. The organic layer was dried over $\mathrm{Na}_{2} \mathrm{SO}_{4}$ and concentrated under reduced pressure. The residue was dissolved in a mixture of petroleum ether and diethylether (9:1) and applied on a short silica column (5g silicagel) to obtain the title compound 3 as a viscous oil in $80 \%$ yield. ${ }^{1} \mathrm{H}$ NMR $\left(\mathrm{CDCl}_{3}, 300 \mathrm{MHz}\right) \delta 5.72-5.78\left(\mathrm{~m}, 1 \mathrm{H}, \mathrm{H}_{2} \mathrm{C}=\mathrm{CH}-\mathrm{CH}_{2}\right)$, 5.14-5.08 $\left(\mathrm{m}, 1 \mathrm{H}, \quad H \mathrm{C}=\mathrm{CH}-\mathrm{CH}_{2}\right), 4.92-4.98(\mathrm{~m}, 1 \mathrm{H}$, $\left.H \mathrm{C}=\mathrm{CH}-\mathrm{CH}_{2}\right), 3.98-4.03\left(\mathrm{~m}, 2 \mathrm{H}, \mathrm{CH}-\mathrm{CH}_{2}-\mathrm{O}\right), 3.32-3.65$ (m, $\left.2 \mathrm{H}, N-\left[\mathrm{CH}\left(\mathrm{CH}_{3}\right)_{2}\right]_{2}\right), 1.01(\mathrm{dd}, J=2.5,10.0 \mathrm{~Hz}, 6 \mathrm{H}$, $\left.\mathrm{CH}\left(\mathrm{CH}_{3}\right)_{2}\right), 0.99\left(\mathrm{dd}, J=2.5,10.5 \mathrm{~Hz}, 6 \mathrm{H}, \mathrm{CH}\left(\mathrm{CH}_{3}\right)_{2}\right)$, -
0.02 (s, 9H, $\left.\mathrm{Si}\left(\mathrm{CH}_{3}\right)_{3}\right) .{ }^{13} \mathrm{C}$ NMR $\left(\mathrm{CDCl}_{3}, 75.5 \mathrm{MHz}\right) \delta$ $135.9\left(\mathrm{~d}, J=5.1 \mathrm{~Hz}, \mathrm{H}_{2} \mathrm{C}=\mathrm{CH}-\mathrm{CH}_{2}\right), 116.2\left(\mathrm{H}_{2} \mathrm{C}=\mathrm{CH}-\right.$ $\left.\mathrm{CH}_{2}\right), 111.0\left(\mathrm{~d},{ }^{3} J_{\mathrm{PC}}=1.8 \mathrm{~Hz}, \mathrm{C}-2\right.$ acetylene $), 107.5(\mathrm{~d}$, ${ }^{2} J_{\mathrm{PC}}=20.3 \mathrm{~Hz}, \mathrm{C}-1$ acetylene $), 67.6\left(\mathrm{~d}, J_{=} 17.1 \mathrm{~Hz}, \mathrm{P}-\right.$ $\left.\mathrm{OCH}_{2}-\mathrm{CH}_{2}\right), 43.1\left(\mathrm{CH}\left(\mathrm{CH}_{3}\right)_{2}\right), 43.3\left(\mathrm{CH}\left(\mathrm{CH}_{3}\right)_{2}\right), 24.4$ $\left(\mathrm{CH}\left(\mathrm{CH}_{3}\right)_{2}\right), 24.9\left(\mathrm{CH}\left(\mathrm{CH}_{3}\right)_{2}\right),-0.0\left(\mathrm{Si}\left(\mathrm{CH}_{3}\right)_{3}\right) .{ }^{31} \mathrm{P} \mathrm{NMR}$ $\left(\mathrm{CDCl}_{3}, 121.5 \mathrm{MHz}{ }^{1} \mathrm{H}\right.$-decoupled) $\delta$ 94.3. MS $\mathrm{m} / z(+\mathrm{v} / \mathrm{e}$, FAB) $[23 \%, \mathrm{M}+\mathrm{H}]^{+} 286,\left[81 \%, \mathrm{M}+\mathrm{H}-\mathrm{C}_{3} \mathrm{H}_{5}\right]^{+} 244,[100 \%$, $\left.\mathrm{M}+\mathrm{H}_{-} \mathrm{C}_{3} \mathrm{H}_{5} \mathrm{O}\right]^{+}$228. HRMS (LSIMS, positive) calcd for $\mathrm{C}_{11} \mathrm{H}_{23} \mathrm{NPSi}\left[\mathrm{M}+\mathrm{H}-\mathrm{C}_{3} \mathrm{H}_{5} \mathrm{O}\right] 228.1337$ found 228.1329

$\beta-5-O$ - Allyl - $1-O$ - methyl - 2,3 - $O, O$ isopropylidene $-\mathrm{D}$ - ribofuranoside $(\mathbf{4})^{21}$

To a solution of D-ribose $(6.30 \mathrm{~g}, 0.042 \mathrm{~mol})$ in $126 \mathrm{~mL}$ methanol, $H$-Dowex resin $(1.4 \mathrm{~g})$ was added. The reaction was stirred $48 \mathrm{~h}$ at room temperature. The $H$-Dowex resin was filtrated off and rinsed portionly with ether. The filtrate was dried over $\mathrm{Na}_{2} \mathrm{SO}_{4}$ and concentrated under reduced pressure. The oil was dissolved in a mixture of acetone $(47.2 \mathrm{~mL})$ and 2,2 dimethoxypropane (0.084 mole, $10 \mathrm{~mL})$. $H$-Dowex resin $(1.4 \mathrm{~g})$ was added and the mixture was stirred overnight. The resin was then removed by filtration and the filtrate evaporated under reduced pressure. $\beta-1-O$ Methyl-2,3-O,O- isopropylidene -D-ribofuranoside ${ }^{21}$ was obtained in quantitative yields as a colourless oil, $R_{f}=0.4$ $\left(\mathrm{CHCl}_{3} /\right.$ Acetone, 9:1) and data match ref. 20. $\mathrm{H}$ NMR $\left(\mathrm{CDCl}_{3}, 500 \mathrm{MHz}\right) \delta 5.05(\mathrm{~s}, 1 \mathrm{H}, \mathrm{H}-1), 4.85(\mathrm{~d}, J=3.9 \mathrm{~Hz}$, $1 \mathrm{H}, \mathrm{H}-2), 4.59$ (d, $J=3.4 \mathrm{~Hz}, 1 \mathrm{H}, \mathrm{H}-3), 4.44$ (t, $J=2.6 \mathrm{~Hz}$, 1H, H-4), 3.6-3.3 (m, 2H, H-5), 3.44 (s, 3H, $\left.\mathrm{OCH}_{3}\right), 1.49$ (s, $3 \mathrm{H}$, isopropylidene- $\left.\mathrm{CH}_{3}\right), 1.32(\mathrm{~s}, 3 \mathrm{H}$, isopropylidene$\left.\mathrm{CH}_{3}\right) . \quad{ }^{13} \mathrm{C} \quad \mathrm{NMR} \quad\left(\mathrm{CDCl}_{3}, \quad 125.75 \quad \mathrm{MHz}\right) \quad \delta 112.1$ (isopropylidene $\left.C\left(\mathrm{CH}_{3}\right)_{2}\right), 110.0(\mathrm{C}-1), 88.8(\mathrm{C}-4), 86.2$ $(\mathrm{C}-2), \quad 81.9$ (C-3), $64.4 \quad(\mathrm{C}-5), \quad 55.9 \quad\left(\mathrm{OCH}_{3}\right), \quad 26.7$ (isopropylidene $-\mathrm{CH}_{3}$ ), 25.0 (isopropylidene- $\mathrm{CH}_{3}$ ).

To a solution of $\beta-1-O$-methyl-2,3-O,O- isopropylidene D-ribofuranoside ( $5 \mathrm{~g}, 0.025$ mole) in $100 \mathrm{~mL} \mathrm{DMF}, \mathrm{BaO}$ $(14.5 \mathrm{~g}, 0.095 \mathrm{~mol})$ and allylbromide $(12.1 \mathrm{~g}, 0.1 \mathrm{~mol})$ were added. The reaction mixture was stirred at rt, overnight. The solution was filtrated through a celite path and rinsed with chloroform. The filtrate was successively washed with water and aq. $\mathrm{KCl}\left(10 \%\right.$, w/v), dried over $\mathrm{Na}_{2} \mathrm{SO}_{4}$ and concentrated to obtain 4 as a colorless oil $(5.5 \mathrm{~g}, 0.023$ mol) in $92 \%$ yield. This compound was used without further purification. ${ }^{1} \mathrm{H}$ NMR $\left(\mathrm{CDCl}_{3}, 300 \mathrm{MHz}\right) \delta .5 .85$ $5.92\left(\mathrm{~m}, 1 \mathrm{H}, \mathrm{H}_{2} \mathrm{C}=\mathrm{C} H-\mathrm{CH}_{2}\right), 5.28 .\left(\mathrm{dd}, J_{\mathrm{gem}}=1.5 \mathrm{~Hz}, J_{\text {trans }}=\right.$ $\left.17.2 \mathrm{~Hz}, 1 \mathrm{H}, \mathrm{H}_{2} \mathrm{C}=\mathrm{CH}-\mathrm{CH}_{2}\right), 5.19\left(\mathrm{dd}, J_{\mathrm{gem}}=1.5 \mathrm{~Hz}, J_{\text {cis }}=\right.$ $\left.10.4 \mathrm{~Hz}, 1 \mathrm{H}, \mathrm{H}_{2} \mathrm{C}=\mathrm{CH}-\mathrm{CH}_{2}\right), 4.96(\mathrm{~s}, 1 \mathrm{H}, \mathrm{H}-1, \beta), 4.68(\mathrm{~d}$, $J=6.0 \mathrm{~Hz}, 1 \mathrm{H}, \mathrm{H}-2), 4.58(\mathrm{~d}, J=6.8 \mathrm{~Hz}, 1 \mathrm{H}, \mathrm{H}-4), 4.02$ $\left(\mathrm{d}, J=5.5 \mathrm{~Hz}, 2 \mathrm{H}, \mathrm{H}_{2} \mathrm{C}=\mathrm{CH}-\mathrm{CH}_{2}\right), 3.41-3.48(\mathrm{~m}, 3 \mathrm{H}, \mathrm{H}-3$, $\mathrm{H}-5), 3.32(\mathrm{~s}, 3 \mathrm{H}, \mathrm{O}-\mathrm{Me}), 1.48$ (s, 3H, isopropylidene$\left.\mathrm{CH}_{3}\right), 1.34$ (s, 3H, isopropylidene- $\left.\mathrm{CH}_{3}\right) \cdot{ }^{13} \mathrm{C} \mathrm{NMR}\left(\mathrm{CDCl}_{3}\right.$, $75.5 \mathrm{MHz}) \delta 136.1\left(\mathrm{H}_{2} \mathrm{C}=\mathrm{CH}-\mathrm{CH}_{2}\right), 117.5\left(\mathrm{H}_{2} \mathrm{C}=\mathrm{CH}-\mathrm{CH}_{2}\right)$, 112.7 (isopro- pylidene- $\left.\mathrm{CH}_{3}\right), 109.6(\mathrm{C}-1), 85.5(\mathrm{C}-4), 82.5$ (C-2), $77.6(\mathrm{C}-3), 72.5\left(\mathrm{H}_{2} \mathrm{C}=\mathrm{CH}-\mathrm{CH}_{2} \mathrm{O}\right), 71.3(\mathrm{C}-5), 55.8$ (O-Me), 26.3 (isopropylidene- $\mathrm{CH}_{3}$ ), 25.3 (isopropylidene $\mathrm{CH}_{3}$ ).

$\alpha / \beta$ 1,2,3-Tri- $O, O, O$-acetyl D-ribofuranose $(\mathbf{5})^{22}$ 
The allylated ribose $4(4.8 \mathrm{~g}, 0.02 \mathrm{~mol})$ was dissolved at $0^{\circ} \mathrm{C}$ in solution containing glacial acetic acid $(7 \mathrm{~mL})$, acetic anhydride $(5 \mathrm{~mL})$ and $\mathrm{H}_{2} \mathrm{SO}_{4}(1.67 \mathrm{~mL})$. The reaction mixture was allowed to warm up progressively and stirred overnight. The solution was poured in a mixture of ice and $\mathrm{NaHCO}_{3}$. The neutralized solution was extracted three times with $\mathrm{CHCl}_{3}$. The organic layer was dried over $\mathrm{Na}_{2} \mathrm{SO}_{4}$, filtered and concentrated. $\alpha / \beta-1,2,3$-Tri- $O, O, O$ acetyl 5-O-allyl D-ribofuranose was obtained as a colourless oil in $75 \%$ yield $(0.015 \mathrm{~mol}, 4.75 \mathrm{~g}) .{ }^{1} \mathrm{H}$ NMR $\left(\mathrm{CDCl}_{3}, 300 \mathrm{MHz}\right) \delta 6.21(\mathrm{~s}, 0.5 \mathrm{H}, \alpha / \beta), 6.15(\mathrm{~s}, 0.5 \mathrm{H}$, $\alpha / \beta), 5.41-5.46$ (m, H-2, $\alpha / \beta)$, 5.32-5.36 (m, H-2, $\alpha / \beta)$, 5.25-5.31 (m, $\left.1 \mathrm{H}, \quad H \mathrm{C}=\mathrm{CH}-\mathrm{CH}_{2}\right), \quad 5.19-5.21(\mathrm{~m}, 1 \mathrm{H}$, $\left.H \mathrm{C}=\mathrm{CH}-\mathrm{CH}_{2}\right), 5.18(\mathrm{~s}, \mathrm{H}-3, \alpha / \beta), 5.13(\mathrm{dd}, J=1.2,5.1$ $\mathrm{Hz}, \mathrm{H}-3, \alpha / \beta)$, 4.29-4.32 (m, 1H, H-4), 4.02-4.07 (m, 2H, $\left.\mathrm{H}_{2} \mathrm{C}=\mathrm{CH}-\mathrm{CH}_{2}\right), \quad 3.60-3.62(\mathrm{~m}, 2 \mathrm{H}, \mathrm{H}-5), 2.07,2 \mathrm{r}_{3} 08,2.10$, $2.11,2.12,2.13,2.14\left(\mathrm{~m}, 9 \mathrm{H}, \mathrm{COOCH}_{3}\right) .{ }^{\mathrm{C}} \mathrm{C} \mathrm{NMR}$ $\left(\mathrm{CDCl}_{3}, 75.47 \mathrm{MHz}\right) \delta 169.3,169.4,169.6,169.8,170.0$ $(\mathrm{C}=\mathrm{O}), \quad 134.3 \quad\left(\mathrm{H}_{2} \mathrm{C}=\mathrm{CH}-\mathrm{CH}_{2}\right), \quad 117.3 \quad\left(\mathrm{H}_{2} \mathrm{C}=\mathrm{CH}-\mathrm{CH}_{2}\right)$, 99.4, $98.3\left(\mathrm{C}_{1}, \alpha / \beta\right), 83.7,81.0\left(\mathrm{C}_{4}, \alpha / \beta\right), 80.9,76.9\left(\mathrm{C}_{3}\right.$, $\alpha / \beta), \quad 74.3,69.6\left(\mathrm{C}_{2}, \quad \alpha / \beta\right), 72.6\left(\mathrm{H}_{2} \mathrm{C}=\mathrm{CH}-\mathrm{CH}_{2}\right), 70.4$, $69.0\left(\mathrm{C}_{5}, \alpha / \beta\right), \quad 20.5,20.7,20.8,21.0,22.5,25.3$ $\left(\mathrm{COOCH}_{3}\right) . \quad \mathrm{MS} m / z(+\mathrm{v} / \mathrm{e}, \mathrm{EI})[5 \%, \mathrm{M}-\mathrm{H}] 315,[100 \%$, $\mathrm{M}-\mathrm{C}_{2} \mathrm{H}_{3} \mathrm{O}_{2}$ ], [64\%, M-2 $\left.\mathrm{xC}_{2} \mathrm{H}_{3} \mathrm{O}_{2}-\mathrm{C}_{2} \mathrm{H}_{3} \mathrm{O}\right]$ 155, [13\%, M$3 \mathrm{xC}_{2} \mathrm{H}_{3} \mathrm{O}_{2}$ ] 139. HRMS (LSIMS, positive) calcd for $\mathrm{C}_{14} \mathrm{H}_{20} \mathrm{O}_{8}$ [M-H] 315.1079 found 315.1077.

$\alpha / \beta$-5-O-Allyl-1, 2, 3- $O, O, O$ - triacetyl D-ribofuranoside $(1.17 \mathrm{~g}, 3.70 \mathrm{mmol})$ was dissolved in a mixture of methanol $(25 \mathrm{~mL})$ and $\mathrm{CH}_{2} \mathrm{Cl}_{2}(25 \mathrm{~mL})$ and the solution was cooled at $0^{\circ} \mathrm{C}$. Palladium chloride $(0.23 \mathrm{~g}, 1.30 \mathrm{mmol})$ was added and the reaction mixture was warmed up to room temperature and vigorously stirred for $4 \mathrm{~h}$. The reaction was quenched with triethylamine $(1.7 \mathrm{~mL}, 12$ mmol) and evaporated under pressure. The residue was dissolved in EtOAc and filtrated through a celite path. The filtrate was concentrated under reduced pressure and the residue was purified by flash chromatography $\left(\mathrm{CHCl}_{3} /\right.$ acetone). Title compound $\mathbf{5}$ was obtained as a yellowish oil in $58 \%(0.60 \mathrm{~g}, 2.16 \mathrm{mmol}$, mixture of $\alpha$ and $\beta$ anomers $)$ and data match ref. $21 .{ }^{1} \mathrm{H}$ NMR $\left(\mathrm{CDCl}_{3}, 300\right.$ MHz) $\delta 6.06(\mathrm{H}-1,0.5 \mathrm{H}, \alpha / \beta), 6.08(\mathrm{H}-1,0.5 \mathrm{H}, \alpha / \beta)$, 5.31-5.33 (m, 0.5 H, H-2, $\alpha / \beta), 5.27(\mathrm{~d}, \mathrm{~J}=4.9 \mathrm{~Hz}, 0.5 \mathrm{H}$, $\mathrm{H}-2, \alpha / \beta), 5.14(\mathrm{~d}, J=1.8 \mathrm{~Hz}, 0.5 \mathrm{H}, \mathrm{H}-3, \alpha / \beta), 5.04(\mathrm{dd}, J$ $=1.8,5.2 \mathrm{~Hz}, 0.5 \mathrm{H}, \mathrm{H}-3, \alpha / \beta), 4.16-4.18(\mathrm{~m}, 1 \mathrm{H}, \mathrm{H}-4)$, 3.71-3.78 (m, 2H, H-5), 2.00, 2.01, 2.03, 2.04, 2.05, 2.06 $\left(\mathrm{m}, 9 \mathrm{H}, \mathrm{COOCH}_{3}\right) .{ }^{13} \mathrm{C} \mathrm{NMR}\left(\mathrm{CDCl}_{3}, 125.75 \mathrm{MHz}\right) \delta$ 169.3, 169.0, 168.9, 168.7, 168.6 (C=O), 98.2, $97.1 \quad\left(\mathrm{C}_{1}\right.$ $\alpha / \beta), 83.8,81.4\left(\mathrm{C}_{4} \alpha / \beta\right), 80.0,76.0\left(\mathrm{C}_{3} \alpha / \beta\right), 73.5,68.9$ $\left(\mathrm{C}_{2} \alpha / \beta\right), 60.8,60.6\left(\mathrm{C}_{5} \alpha / \beta\right), 20.0,19.8,19.7,19.6,19.5$, 19.4, $19.3\left(\mathrm{COOCH}_{3}\right)$. HRMS (LSIMS, positive) calcd for $\mathrm{C}_{11} \mathrm{H}_{16} \mathrm{O}_{8}[\mathrm{M}-\mathrm{OH}]^{+} 259.0817$ found 259.0830 .

2, 3- $O, O$-Triacetyl-5-O-[(allyl ethynephosphonyl]-Dribofuranoside $(\mathbf{6})$

To a solution of $5(0.60 \mathrm{~g}, 2.10 \mathrm{mmol})$ and $\mathbf{3}(0.58 \mathrm{~g}, 2.10$ mmol) in acetonitrile $(4.5 \mathrm{~mL})$ was added drop wise 2,4 - dinitrophenol $(0.58 \mathrm{~g}, 3.15 \mathrm{mmol})$ dissolved in acetonitrile $(3 \mathrm{~mL})$. The reaction was stirred for $2 \mathrm{~h}$, diluted with 160 $\mathrm{mL}$ of $\mathrm{CH}_{2} \mathrm{Cl}_{2}$ and cooled down to $0^{\circ} \mathrm{C}$. The oxidation was carried out by addition of aq- $\mathrm{H}_{2} \mathrm{O}_{2}(30 \%, \mathrm{w} / \mathrm{v}, 2.5 \mathrm{~mL}$, $12.40 \mathrm{mmol}$ ). The reaction was stirred for an additional 7 min at $0^{\circ} \mathrm{C}$ then quenched with $355 \mathrm{~mL}$ of a saturated $\mathrm{NaHCO}_{3}$ solution. The aqueous layer was extracted with $\mathrm{CH}_{2} \mathrm{Cl}_{2}$ and the combined organic layers were dried over $\mathrm{Na}_{2} \mathrm{SO}_{4}$. The solvent was removed under reduced pressure and the oily residue $(0.75 \mathrm{~g}, 1.58 \mathrm{mmol})$ was dissolved in dry ethanol $(7.5 \mathrm{~mL})$ containing $\mathrm{CsF}(0.30 \mathrm{~g}, 1.58 \mathrm{mmol})$. The reaction mixture was stirred for $1 \mathrm{~h}$. After addition of a saturated solution of $\mathrm{NaHCO}_{3}$, the aqueous layer was extracted three times with $\mathrm{CH}_{2} \mathrm{Cl}_{2}$. The combined organic layers were dried over $\mathrm{Na}_{2} \mathrm{SO}_{4}$ and concentrated under reduced pressure. The oily residue was purified by flash chromatography $\left(\mathrm{CHCl}_{3} / \mathrm{CH}_{3} \mathrm{OH}: 97.5 / 2.5\right.$, v/v) to obtain 6 as colourless oil in $50 \%$ yield ( 3 steps). ${ }^{1} \mathrm{H}$ NMR $\left(\mathrm{CDCl}_{3}\right.$, $300 \mathrm{MHz}) \delta 6.14(\mathrm{~s}, 0.6 \mathrm{H}, \beta$-anomer, H-1'), 6.08 (s, $0.4 \mathrm{H}$, $\alpha$-anomer, $\left.\mathrm{H}-1^{\prime}\right), 5.85-5.90\left(\mathrm{~m}, 1 \mathrm{H}, \mathrm{H}_{2} \mathrm{C}=\mathrm{CH}-\mathrm{CH}_{2}\right), 5.34$ $\left(\mathrm{dd}, J=17.2 \mathrm{~Hz}, 1.2 \mathrm{~Hz}, 1 \mathrm{H}, H \mathrm{C}=\mathrm{CH}-\mathrm{CH}_{2}\right), 5.24-5.26(\mathrm{~m}$, $\left.1 \mathrm{H}, \mathrm{H}-2^{\prime}\right), 5.22(\mathrm{dd}, J=10.3 \mathrm{~Hz}, 0.8 \mathrm{~Hz}, 1 \mathrm{H}, H \mathrm{C}=\mathrm{CH}-$ $\left.\mathrm{CH}_{2}\right), 5.13\left(\mathrm{~d}, J=1.3 \mathrm{~Hz}, \mathrm{H}, \mathrm{H}-3{ }^{\prime}, \alpha / \beta\right), 5.02(\mathrm{~d}, J=3.0$ $\mathrm{Hz}, \mathrm{H}, \alpha / \beta), 4.56-4.59\left(\mathrm{~m}, 2 \mathrm{H}, \mathrm{H}_{2} \mathrm{C}=\mathrm{CH}-\mathrm{CH}_{2}\right), 4.13-4.29$ $\left(\mathrm{m}, 3 \mathrm{H}, \mathrm{H}-4^{\prime} \& \mathrm{H}-5^{\prime}\right), 2.92\left(\mathrm{~d}, J_{\mathrm{PH}}=13.6 \mathrm{~Hz}, 1 \mathrm{H}, H-\right.$ acetylene), 1.99, 2.00, 2.01, 2.04, 2.05, 2.06 ( 9H, $\left.\mathrm{COOCH}_{3}\right) .{ }^{13} \mathrm{C} \mathrm{NMR}\left(\mathrm{CDCl}_{3}, 75.5 \mathrm{MHz}\right) \delta 169.6,169.8$, $169.9,170.1,170.4,170.6(\mathrm{C}=\mathrm{O}), 132.1\left(\mathrm{~d},{ }^{4} J_{\mathrm{PC}}=7.2 \mathrm{~Hz}\right.$, $\left.\mathrm{H}_{2} \mathrm{C}=C \mathrm{H}-\mathrm{CH}_{2}\right), 119.37\left(\mathrm{~d},{ }^{5} J_{\mathrm{PC}}=4.4 \mathrm{~Hz}, \mathrm{H}_{2} \mathrm{C}=\mathrm{CH}-\mathrm{CH}_{2}\right)$, 99.74 (C-1' $\beta$ anomer), 98.3 (C-1' $\alpha$ anomer), 89.3 (d, ${ }^{2} J_{\mathrm{PC}}=$ $52.5 \mathrm{~Hz}, \mathrm{C}-6$ acetylene), $89.1\left(\mathrm{~d},{ }^{2} J_{\mathrm{PC}}=52.3 \mathrm{~Hz}, \mathrm{C}-6\right.$ acetylene), 83.5 (dd , ${ }^{4} J_{\mathrm{PC}}=8.1,2.5 \mathrm{~Hz}, \mathrm{C}-4$ ' $, \alpha / \beta, \mathrm{R}$ or $\mathrm{S}$ ), $80.8\left(\mathrm{C}-3{ }^{\prime}, \alpha / \beta\right), 80.0\left(\mathrm{dd},{ }^{3} J_{\mathrm{PC}}=8.2,5.0 \mathrm{~Hz}, \mathrm{C}-4{ }^{\prime}, \alpha / \beta, \mathrm{R}\right.$ or S), $77.7\left(\mathrm{C}-3^{\prime}, \alpha / \beta\right),, 74.6\left(\mathrm{C}-2^{\prime}, \alpha / \beta\right), 73.9\left(\mathrm{~d},{ }^{3} J_{\mathrm{PC}}=\right.$ $297.5 \mathrm{~Hz}, \mathrm{C}-7$ acetylene $), 70.5(\mathrm{C}-2, \alpha / \beta), 68.2\left(\mathrm{~d},{ }^{3} \mathrm{~J}_{\mathrm{PC}}=\right.$ $\left.5.1 \mathrm{~Hz}, \mathrm{H}_{2} \mathrm{C}=\mathrm{CH}-\mathrm{CH}_{2}\right), 65.8\left(\mathrm{~d},{ }^{3} J_{\mathrm{PC}}=3.3 \mathrm{~Hz}, \mathrm{C}-5{ }^{\prime}, \alpha / \beta\right)$, $65.78\left(\mathrm{~d},{ }^{3} J_{\mathrm{PC}}=4.8 \mathrm{~Hz}, \mathrm{C}-5\right.$ ', $\left.\alpha / \beta\right), 20.8,20.9,21.0,21.3$, 21.4, $21.6\left(\mathrm{COOCH}_{3}\right) .{ }^{31} \mathrm{P}$ NMR $\left(\mathrm{CDCl}_{3}, 121.5 \mathrm{MHz}{ }^{1} \mathrm{H}-\right.$ decoupled) $\delta-7.28(\mathrm{~d}, J=4.5 \mathrm{~Hz}, \alpha / \beta),-7.39(\mathrm{~d}, J=3.4$ $\mathrm{Hz}, \alpha / \beta)$. MS $m / z$ (+ v/e, Electrospray) $[\mathrm{M}+\mathrm{Na}]^{+}$427.1. MS $\mathrm{m} / \mathrm{z}(+\mathrm{v} / \mathrm{e}, \mathrm{FAB}),\left[100 \%, \quad \mathrm{M}-\mathrm{C}_{2} \mathrm{H}_{3} \mathrm{O}_{2}\right]$ 345. HRMS (LSIMS, positive) calcd for $\mathrm{C}_{13} \mathrm{H}_{14} \mathrm{O}_{9} \mathrm{P} \quad\left[\mathrm{M}-\mathrm{C}_{2} \mathrm{H}_{3} \mathrm{O}_{2}\right]$ 345.0375 found 345.0359 .

$\mathrm{P}^{1}$-[Allyloxy-diisopropylamino]- $\mathrm{P}^{2}-[(\alpha / \beta-1,2,3-O, O, O-$ triacetyl)-D-ribofuranosid-5-yl-allyl] -acetylene- (1phosphine, 2-phosphonate) (7)

To a THF solution $(5.7 \mathrm{~mL})$ of $6(0.22 \mathrm{~g}, 0.54 \mathrm{mmol})$ and diisopropylaminochlorophosphine $(0.12 \mathrm{~g}, 0.54 \mathrm{mmol})$ cooled at $-78^{\circ} \mathrm{C}$ was added $n$-butyllithium ( $2 \mathrm{M}$ in hexane, $0.33 \mathrm{~mL}, 0.60 \mathrm{mmol}$ ). The solution was stirred for $50 \mathrm{~min}$ at $-78^{\circ} \mathrm{C}$ and quenched with a mixture of $\mathrm{CH}_{2} \mathrm{Cl}_{2}$ and saturated $\mathrm{NaHCO}_{3}$ solution $(17 \mathrm{~mL} / 12 \mathrm{~mL})$. The aqueous layer was extracted three times with $\mathrm{CH}_{2} \mathrm{Cl}_{2}$. The combined organic layers were dried over $\mathrm{Na}_{2} \mathrm{SO}_{4}$ and concentrated under reduced pressure. The residue was dissolved in a mixture of diethylether-pentane (3/1) and applied on a short silica column (diethylether/pentane (3/1) 
used as eluant) to obtain the title compound $\mathbf{7}$ as a colorless oil in $53 \%$ yield. ${ }^{1} \mathrm{H}$ NMR $\left(\mathrm{CDCl}_{3}, 300 \mathrm{MHz}\right) 6.13(\mathrm{~s}, 1 \mathrm{H}$, $2 / 3 \mathrm{H}-1,2 \beta$-anomer), 6.08 (s, $1 \mathrm{H}, 1 / 3 \mathrm{H}-1$ ', $\alpha$-anomer), 5.84-5.87 (m, 2H, $\left.\mathrm{H}_{2} \mathrm{C}=\mathrm{CH}-\mathrm{CH}_{2}\right), 5.31-5.34(\mathrm{~m}, 2 \mathrm{H}$, $\left.\mathrm{H}_{2} \mathrm{C}=\mathrm{CH}-\mathrm{CH}_{2}\right), 5.23-5.28\left(\mathrm{~m}, 1 \mathrm{H}, \mathrm{H}-2{ }^{\prime}\right), 5.12-5.21(\mathrm{~m}, 1 \mathrm{H}$, $\left.H \mathrm{C}=\mathrm{CH}-\mathrm{CH}_{2}\right), 5.08-5.1 \quad\left(\mathrm{~m}, 1 \mathrm{H}, \quad H \mathrm{C}=\mathrm{CH}-\mathrm{CH}_{2}\right), 5.03 \&$ $5.13\left(\mathrm{~s}, 1 \mathrm{H}, \mathrm{H}-3^{\prime}, \alpha / \beta\right), 4.53-4.55\left(\mathrm{~m}, 2 \mathrm{H}, \mathrm{H}_{2} \mathrm{C}=\mathrm{CH}-\mathrm{CH}_{2}\right)$, 4.31-4.32 (m, 1H, H-4'), 4.25-4.26 (m, 2H, H-5'), 4.13$4.15\left(\mathrm{~m}, 2 \mathrm{H}, \mathrm{H}_{2} \mathrm{C}=\mathrm{CH}-\mathrm{CH}_{2}\right), 3.62\left(\mathrm{~m}, 2 \mathrm{H}, \mathrm{CH}\left(\mathrm{CH}_{3}\right)_{2}\right)$, $2.00,2.01,2.02,2.03,2.04,2.05,2.06\left(9 \mathrm{H}, \mathrm{COOCH}_{3}\right)$, $1.15\left(\mathrm{~d}, J=6.7 \mathrm{~Hz}, 6 \mathrm{H}, \mathrm{CH}\left(\mathrm{CH}_{3}\right)_{2}\right), 1.13(\mathrm{~d}, J=6.8 \mathrm{~Hz}$, $\left.6 \mathrm{H}, \mathrm{CH}\left(\mathrm{CH}_{3}\right)_{2}\right) .{ }^{13} \mathrm{C} \mathrm{NMR}\left(\mathrm{CDCl}_{3}, 75.5 \mathrm{MHz}\right) \delta 169.6$, $169.8,170.0,170.2,170.4(\mathrm{C}=\mathrm{O}), 135.4\left(\mathrm{~d},{ }^{4} J_{\mathrm{PC}}=7.2 \mathrm{~Hz}\right.$, $\left.\mathrm{H}_{2} \mathrm{C}=\mathrm{CH}-\mathrm{CH}_{2}\right), 132.7\left(\mathrm{~d},{ }^{4} \mathrm{~J}=7.3 \mathrm{~Hz}, \mathrm{H}_{2} \mathrm{C}=\mathrm{CH}-\mathrm{CH}_{2}\right), 119.0$ $\left(\mathrm{d},{ }^{5} \mathrm{~J}=8.6 \mathrm{~Hz}, \mathrm{H}_{2} \mathrm{C}=\mathrm{CH}-\mathrm{CH}_{2}\right), 117.6\left(\mathrm{H}_{2} \mathrm{C}=\mathrm{CH}-\mathrm{CH}_{2}\right)$, $104.8\left(\mathrm{~d},{ }^{2} J_{\mathrm{P}(\mathrm{III})-\mathrm{C}}=39 \mathrm{~Hz}, \mathrm{P}(\mathrm{V})-\mathrm{C} C\right.$-P(III)), $99.9\left(\mathrm{C}-1^{\prime}, \beta-\right.$ anomer), 98.5 (C-1', $\alpha$-anomer), $91.3\left(\mathrm{~d},{ }^{2} J_{\mathrm{P}(\mathrm{V})-\mathrm{C}}=286.8 \mathrm{~Hz}\right.$ $\mathrm{P}(\mathrm{V})-C \mathrm{C}-\mathrm{P}(\mathrm{III})), 83.5\left(\mathrm{dd},{ }^{4} J_{\mathrm{PC}}=8.1,4.0 \mathrm{~Hz}, \mathrm{C}-4\right.$ ', $\alpha / \beta, \mathrm{R}$ or S), $80.77(\mathrm{C}-3$ ', $\alpha / \beta), 80.73\left(\mathrm{C}-3{ }^{\prime}, \alpha / \beta\right), 80.2\left(\mathrm{~d},{ }^{4} J_{\mathrm{PC}}=\right.$ $\left.\left.\left.8.5 \mathrm{~Hz}, \mathrm{C}-4{ }^{\prime}, \alpha / \beta\right), 74.5\left(\mathrm{C}-2^{\prime}, \alpha / \beta\right)\right), 70.6\left(\mathrm{C}-2^{\prime}, \alpha / \beta\right)\right), 68.4$ $\left(\mathrm{d},{ }^{3} J_{\mathrm{PC}}=20.0 \mathrm{~Hz}, \mathrm{H}_{2} \mathrm{C}=\mathrm{CH}-\mathrm{CH}_{2}\right), 67.9\left(\mathrm{~d},{ }^{3} J_{\mathrm{PC}}=9.1 \mathrm{~Hz}\right.$, $\left.\mathrm{H}_{2} \mathrm{C}=\mathrm{CH}-\mathrm{CH}_{2}\right), 65.7\left(\mathrm{C}-5{ }^{\prime}, \alpha / \beta\right), 65.57,65.51,65.45\left(\mathrm{C}-5{ }^{\prime}\right.$, $\alpha / \beta) 44.1\left(\mathrm{CH}\left(\mathrm{CH}_{3}\right)_{2}\right), 21.9\left(\mathrm{CH}\left(\mathrm{CH}_{3}\right)_{2}\right), 19.4,19.5,19.7$, 20.0, $20.1\left(\mathrm{CH}_{3}\right.$-acetate $){ }^{31} \mathrm{P} \mathrm{NMR}\left(\mathrm{CDCl}_{3}, 121.5 \mathrm{MHz}{ }^{1} \mathrm{H}-\right.$ decoupled) $\delta+96.5(\mathrm{~d}, J=6.7 \mathrm{~Hz}, \alpha / \beta),+96.3(\mathrm{~d}, J=8.4$ $\mathrm{Hz}, \alpha / \beta),-6.60,-6.66,-6.70,-6.73,-6.77,-6.83$. MS $m / z(+$ v/e, FAB) $[100 \%, \mathrm{M}+\mathrm{H}]^{+}$592, [14\%, M- $\left.\mathrm{C}_{2} \mathrm{H}_{3} \mathrm{O}\right] 548$, $\left[52 \%, \mathrm{M}-\mathrm{C}_{2} \mathrm{H}_{3} \mathrm{O}_{2}\right]$ 532, [28\%, M- $\left.\mathrm{C}_{6} \mathrm{H}_{14} \mathrm{~N}\right]$ 492, [41\%, M$\mathrm{C}_{14} \mathrm{H}_{24} \mathrm{P}_{2} \mathrm{~N}$ ], 276. HRMS (LSIMS, positive) calcd for $\mathrm{C}_{25} \mathrm{H}_{39} \mathrm{NO}_{11} \mathrm{P}_{2}[\mathrm{M}+\mathrm{H}]^{+}$592.2076 found 592.2102.

$\mathrm{P}^{1}$-[Allyl $\quad\left(2^{\prime}, 3^{\prime}-O\right.$-isopropylidene $)$-adenosin-5'-yl]- $\mathrm{P}^{2}$ [allyl $(\alpha / \beta-1 ", 2 ", 3 "-O, O, O$-triacetyl)-D-ribofuranosid-5"yl]-acetylene-bis(phosphonate) (8).

The aminophosphine 7 (0.34 g, $0.57 \mathrm{mmol})$ was dissolved with 2',3'-O,O-isopropylidene adenosine $(0.17 \mathrm{~g}$, $0.57 \mathrm{mmol})$ in acetonitrile $(23 \mathrm{~mL})$ and stirred for $10 \mathrm{~min}$ at rt. To this mixture, 2,4-DNP $(0.157 \mathrm{~g}, 0.855 \mathrm{mmol})$ dissolved in $0.95 \mathrm{~mL}$ of acetonitrile was added dropwise. After 5h, an additional equivalent of 2,4-DNP and of 2',3'$O, O$-isopropylidene adenosine were added. The reaction was stirred overnight and then cooled to $-40^{\circ} \mathrm{C}$. $t$ - $\mathrm{BuOOH}$ (solution 5-6 $\mathrm{M}$ in decane, $0.17 \mathrm{~mL}, 0.85 \mathrm{mmol}$ ) was added and the reaction was stirred for $7 \mathrm{~min}$. A mixture of $\mathrm{CH}_{2} \mathrm{Cl}_{2}$ and a saturated $\mathrm{NaHCO}_{3}$ solution $(50: 30 \mathrm{~mL}$, v/v) was added and the aqueous layer was extracted with $\mathrm{CH}_{2} \mathrm{Cl}_{2}$ $(3 \times 20 \mathrm{~mL})$. The combined organic layers were dried over $\mathrm{Na}_{2} \mathrm{SO}_{4}$ and concentrated under reduced pressure. The oily residue was chromatographed on silica gel (pentane/diethylether; 1/3; v/v) to obtain the bisphosphonate 8 as a colorless oil $(0.145 \mathrm{~g})$ in $31 \%$ yield (over two steps). ${ }^{1} \mathrm{H}$ NMR $\left(\mathrm{CDCl}_{3}, 500 \mathrm{MHz}\right) \delta 8.34$ (s, 1 $\mathrm{H}, \mathrm{H}-2$ ade), 7.99-8.03 (m, 1H, H-8 ade), 6.38 (bs, $2 \mathrm{H}$, $\mathrm{NH}_{2}$ ade), 6.23 (s, $0.6 \mathrm{H}, \mathrm{H}-1$ ", $\beta$-anomer), 6.16 (s, $0.4 \mathrm{H}$, H-1", $\alpha$-anomer) $6.15(\mathrm{~s}, 1 \mathrm{H}, \mathrm{H}-1$ '), 5.80-6.0 (m, 2H, $\left.\mathrm{H}_{2} \mathrm{C}=\mathrm{CH}-\mathrm{CH}_{2} \mathrm{O}\right), 5.42-5.44(\mathrm{~m} ., 1 \mathrm{H}, \mathrm{H}-2$ ) $)$ 5.33-5.42 (m, $1 \mathrm{H}, \mathrm{H}$ ), 5.21-5.33 (m, 5H, $\left.\mathrm{H}_{2} \mathrm{C}=\mathrm{CH}-\mathrm{CH}_{2} ; \mathrm{H}-2 "\right), 5.20$ (bs, H-3", $\alpha / \beta), 5.15-5.17$ (m, 1H, H-3'), 5.13 (bs, H-3", $\alpha / \beta$ ),
4.63-4.65 (m, 2H, $\left.\mathrm{H}_{2} \mathrm{C}=\mathrm{CH}-\mathrm{CH}_{2}\right), 4.55-4.57(\mathrm{~m}, 2 \mathrm{H}$, $\left.\mathrm{H}_{2} \mathrm{C}=\mathrm{CH}-\mathrm{CH}_{2}\right), 4.50-4.54(\mathrm{~m}, 1 \mathrm{H}, \mathrm{H}-4$ '), 4.39-4.45 (m, $2 \mathrm{H}$, H-5'), 4.28-4.38 (m, 3H, H-4"\& H-5"), 2.09, 2.10, 2.105, $2.11,2.115,2.121,2.126,2.127,2.128,2.132,2.138$ $\left(\mathrm{COOCH}_{3}\right), 1.62$ (s, $3 \mathrm{H}, \mathrm{CH}_{3}$-isopropylidene), 1.41 (s, 3H, $\mathrm{CH}_{3}$ isopropylidene). ${ }^{13} \mathrm{C} \mathrm{NMR}\left(\mathrm{CDCl}_{3}, 75.5 \mathrm{MHz}\right) 169.5$, $169.8,170.0,170.5,170.8 \quad(\mathrm{C}=\mathrm{O}), 155.6$ (C-6 ade), 153.0 (C-2 ade), 149.0 (C-4 ade), 139.7 (C-8 ade), 131.2 (d, $J=6.2 \mathrm{~Hz}, \mathrm{H}_{2} \mathrm{C}=\mathrm{CH}-\mathrm{CH}_{2}$ ), 119.8 (C-5 ade), 119.5 $\left(\mathrm{H}_{2} \mathrm{C}=\mathrm{CH}-\mathrm{CH}_{2}\right), 114.5$ (C-isopropylidene), 99.2 (C-1", $\beta$ anomer), 97.8 (C-1", $\alpha$ anomer) , 90.7 (d, $J=8.1 \mathrm{~Hz}, \mathrm{C}-$ $\left.1^{\prime}\right)$, 89.35, 89.6, 89.75, 89.9, 90.1, $\left(\mathrm{P}^{1}-\mathrm{CC}-\mathrm{P}^{2}\right), 85.9,86.2$, $86.5\left(\mathrm{P}^{1}-\mathrm{CC}-\mathrm{P}^{2}\right), 85.0(\mathrm{~d}, J=5.4 \mathrm{~Hz}, \mathrm{C}-4$ ', $\alpha / \beta), 84.0(\mathrm{C}-$ $\left.2^{\prime}\right), 82.9(\mathrm{~d}, J=5.5 \mathrm{~Hz}, \mathrm{C}-4 "), 81.2\left(\mathrm{~d}, J=3.5 \mathrm{~Hz}, \mathrm{C}-3^{\prime}\right)$, $80.2(\mathrm{~d}, J=3.0 \mathrm{~Hz}, \mathrm{C}-4 ", \alpha / \beta), 78.6(\mathrm{C}-3 ", \alpha / \beta), 77.2(\mathrm{C}-3 "$, $\alpha / \beta), 74.0(\mathrm{~d}, J=7.1 \mathrm{~Hz}, \mathrm{C}-2 ", \alpha / \beta), 69.8(\mathrm{~d}, J=7.7 \mathrm{~Hz}, \mathrm{C}-$ 2", $\alpha / \beta), 68.5\left(\mathrm{H}_{2} \mathrm{C}=\mathrm{CH}-\mathrm{CH}_{2}\right), 66.9$ (bs, C5'), 66.0, 66.1, 66.4, 66.8 (C-5"), $27.5\left(\mathrm{CH}_{3}\right.$-isopropylidene), $25.19\left(\mathrm{CH}_{3^{-}}\right.$ isopropylidene), 20.3, 20.4, 20.5, 20.9, $\left(\mathrm{COOCH}_{3}\right) .{ }^{31} \mathrm{P}$ NMR $\left(\mathrm{CDCl}_{3}, 121.5 \mathrm{MHz}{ }^{1} \mathrm{H}\right.$-decoupled) $\delta$ from -9.41 to 9.86, multiplet (9 peaks: $-9.41,-9.48,-9.54,-9.57,-9.63$, $9.64,-9.67,-9.74,-9.77,-9.83,-9.86)$. MS $m / z(+$ v/e, FAB) $[100 \%, \mathrm{M}+\mathrm{H}]^{+} 814,\left[10 \%, \mathrm{M}-\mathrm{C}_{2} \mathrm{H}_{3} \mathrm{O}_{2}\right]^{+} 754,[48 \%$, M- $\left.\mathrm{C}_{19} \mathrm{H}_{25} \mathrm{P}_{2} \mathrm{O}_{13}\right]^{+} 290$. HRMS (LSIMS, positive) calcd for $\mathrm{C}_{32} \mathrm{H}_{42} \mathrm{O}_{16} \mathrm{~N}_{5} \mathrm{P}_{2}[\mathrm{M}+\mathrm{H}]^{+} 814.2098$ found 814.2085.

Allyl (2', 3'-O, $O$-isopropylidene)- D-adenosin-5'-ylmethyl phosphate (10)

Compound 8 (0.010 g, $0.0123 \mathrm{mmol})$ was dissolved in 2.5 $\mathrm{mL}$ methanol and added to a cooled (ice bath) methanol solution saturated with ammonia. Gaseous ammonia was bubbled through this solution during $5 \mathrm{~min}$ and the mixture was stirred for $1 \mathrm{~h}$ at RT. The solvent was removed under vacuum and the residue was dissolved in a minimum of chloroform and applied on a preparative TLC using as eluant a mixture of chloroform and methanol (10:1). Compound 10 was obtained in $55 \%$ yield $(0.003 \mathrm{~g}, 0.0068$ mmol). ${ }^{1} \mathrm{H}$ NMR $\left(\mathrm{CDCl}_{3}, 300 \mathrm{MHz}\right) \delta 8.36(\mathrm{~s}, 1 \mathrm{H}, \mathrm{H}-2$ ade), 8.02 (s, 1H, H-8 ade), 6.14 (d, $J=2.3 \mathrm{~Hz}, \mathrm{H} 1$-ade), 5.79-5.92 (m, $\left.1 \mathrm{H}, \mathrm{CH}_{2}=\mathrm{CHCH}_{2} \mathrm{O}\right), 5.70$ (bs, $2 \mathrm{H}, \mathrm{NH}_{2}$-ade), 5.40-5.43 (m, 1H, H-2' ade), 5.23-5.34 (m, 2H, $\mathrm{CH}_{2}=\mathrm{CHCH}_{2} \mathrm{O}$ ), 5.10-5.12 (m, $1 \mathrm{H}, \mathrm{H}-3$ ' ade), 4.44-4.51 (m, 3H, $\mathrm{CH}_{2}=\mathrm{CHCH}_{2} \mathrm{O}, \mathrm{H}-4$ ' ade), 4.20-4.25 (m, 2H, H-5' ade), 1.62 (s, $3 \mathrm{H}, \mathrm{CH}_{3}$-acetonide). ${ }^{31} \mathrm{P} \mathrm{NMR}\left(\mathrm{CDCl}_{3}, 121.5\right.$ $\mathrm{MHz}{ }^{1} \mathrm{H}$-decoupled) $\delta 0.62,0.65 \mathrm{ppm}$. MS $\mathrm{m} / \mathrm{z}$ (+ ve Electrospray) $[100 \% \mathrm{M}+\mathrm{H}]^{+} 442$ and $[15 \% \mathrm{M}+\mathrm{Na}]^{+} 464$ $\left(\mathrm{C}_{17} \mathrm{H}_{24} \mathrm{O}_{7} \mathrm{~N}_{5} \mathrm{P}\right.$ requires $\left.M, 441\right)$. MS $m / z$ (+v/e, FAB) $[38 \%, \mathrm{M}+\mathrm{H}]^{+} 442,\left[100 \%, \mathrm{M}-\mathrm{C}_{5} \mathrm{H}_{4} \mathrm{~N}_{5}\right]$ 307, [23\%, M+H$\left.\mathrm{C}_{5} \mathrm{H}_{4} \mathrm{~N}_{5}\right]^{+}$308. HRMS (LSIMS, positive) calcd for $\mathrm{C}_{17} \mathrm{H}_{25} \mathrm{O}_{7} \mathrm{~N}_{5} \mathrm{P}[\mathrm{M}+\mathrm{H}]^{+} 442.1491$ found 442.1507 .

$\mathrm{P}^{1}$-[Allyl-adenosin-5'-yl]-P $\mathrm{P}^{2}$-[allyl $\quad(\alpha / \beta-1 ", 2$ ",3"- $O, O, O$ triacetyl)-D-ribofuranosid-5"-yl]-acetylenebis(phosphonate) (9).

Compound 8 (0.0195 g, $0.024 \mathrm{mmol})$ was dissolved in a mixture of water $(0.40 \mathrm{~mL})$ and TFA $(0.76 \mathrm{~mL})$. The solution was stirred for $40 \mathrm{~min}$ at RT and evaporated under 
high vacuum. Residual TFA was removed by coevaporating three times with toluene. The residue was dissolved in minimum of chloroform and applied on a preparative TLC using as eluent a mixture of chloroform/methanol (10:1) to obtain the title compound as colourless oil in $65 \%$ yield. Unreacted starting material (4.5 mg, 23\%) was recovered. ${ }^{1} \mathrm{H}$ NMR $\left(\mathrm{CDCl}_{3}, 300\right.$ MHz) $\delta 8.19$ (s, $1 \mathrm{H}, \mathrm{H}-2$ ade), $8.00(\mathrm{~m}, 1 \mathrm{H}, \mathrm{H}-8 \mathrm{ade})$, 6.07 ( s, $0.6 \mathrm{H}, \mathrm{H}-1$ ", $\beta$-anomer), 6.06 (s, $0.4 \mathrm{H}, \mathrm{H}-1$ ", $\alpha$ anomer), 5.97 (d, $\left.J=4.7 \mathrm{~Hz}, 1 \mathrm{H}, \mathrm{H}-1^{\prime}\right), 5.72-5.92(\mathrm{~m}, 2 \mathrm{H}$, $\mathrm{H}_{2} \mathrm{C}=\mathrm{CH}-\mathrm{CH}_{2} \mathrm{O}$ ), 5.35-5.36 (m., $1 \mathrm{H}, \mathrm{H}-2$ ) , 5.21-5.33 (m, $6 \mathrm{H}, \mathrm{H}_{2} \mathrm{C}=\mathrm{CH}-\mathrm{CH}_{2} ; \mathrm{m}, 1 \mathrm{H}, \mathrm{H}-2^{\prime \prime} ; \mathrm{m}, 1 \mathrm{H}, \mathrm{H}-3$ '), 5.19 (bs, $1 \mathrm{H}, \mathrm{H}-3 ", 0.6 \beta$ ), 5.18 (bs, $1 \mathrm{H}, \mathrm{H}-3 ", 0.4 \alpha$ ), 4.53-4.61 (m, $\left.4 \mathrm{H}, \mathrm{H}_{2} \mathrm{C}=\mathrm{CH}-\mathrm{CH}_{2}\right), 4.50-4.52(\mathrm{~m}, 2 \mathrm{H}, \mathrm{H}-5)$, 4.27-4.42 (m, 4H, H-4', H-4"\& H-5"), 3.72 ( dd, $J=4.35,11.25 \mathrm{~Hz}, 3 \mathrm{H}$, $\left.\mathrm{POCH}_{3}\right), 1.99,2.00,2.01,2.03,2.04,2.047,2.05,2.06$, $2.07\left(\mathrm{COOCH}_{3}\right) .{ }^{31} \mathrm{P}$ NMR $\left(\mathrm{CDCl}_{3}, 121.5 \mathrm{MHz}{ }^{1} \mathrm{H}-\right.$ decoupled) $\delta-8.50,-8.52 \mathrm{ppm}$. MS $\mathrm{m} / z$ (+ ve Electrospray) $[\mathrm{M}+\mathrm{H}]^{+} \quad 774$ and $[\mathrm{M}+\mathrm{Na}]^{+} 796$ $\left(\mathrm{C}_{29} \mathrm{H}_{37} \mathrm{~N}_{5} \mathrm{O}_{16} \mathrm{P}_{2}\right.$ requires $\left.M, 773\right)$

$\mathrm{P}^{1}$-[(Adenosin-5'-yl]-P2-[ $\alpha / \beta$-D-ribofuranosid-5"-yl]acetylene-bis(phosphonate) (2).

Compound 9 (0.012 g, $0.0157 \mathrm{mmol}), \mathrm{PPh}_{3}(0.0012 \mathrm{~g}$, $0.0046 \mathrm{mmol})$, formic acid $(0.0017 \mathrm{~mL}, 0.045 \mathrm{mmol})$ and triethylamine $(0.007 \mathrm{~mL}, 0.043 \mathrm{mmol})$ were dissolved in $0.2 \mathrm{~mL}$ THF. To this solution, $\mathrm{Pd}\left[\mathrm{PPh}_{3}\right](0.003 \mathrm{~g}, 0.0026$ $\mathrm{mmol}$ ) was added and the resulting solution was stirred for $8 \mathrm{~h}$ at RT and the solution was evaporated under reduced pressure. The residue was dissolved in $0.5 \mathrm{~mL}$ water and three times extracted with $0.5 \mathrm{~mL}$ diethylether. The aqueous layer was evaporated under high vacuum and the residue was dried by coevaporating three times with toluene then dissolved in $0.5 \mathrm{~mL}$ of a $0.1 \mathrm{M}$ Sodium methoxide in methanol solution $(0.05 \mathrm{mmol})$ and vigorously stirred at RT for $25 \mathrm{~min}$. The solution was quenched with a formate solution $(50 \mathrm{mM}$ formic acid, $\mathrm{pH}=3$ with ammonia solution) and the resulting solution evaporated under high vacuum. The residue was purified by reversed phase column chromatography (method 2 ) and the fractions containing the title compound were evaporated under high vacuum. The excess of ammonium salts were removed by coevaporating several times with methanol. 2 (0.006 g, 0.0106 mole) was obtained in $67 \%$ yield. ${ }^{1} \mathrm{H}$ NMR $\left(\mathrm{D}_{2} \mathrm{O}, 300 \mathrm{Mhz}\right) \delta 8.31$ (s, $1 \mathrm{H}, \mathrm{H}-2$ ade), 8.16 (s, 1H, H-8 ade), 5.83 (d, $\left.J=11.4 \mathrm{~Hz}, 1 \mathrm{H}, \mathrm{H}-1^{\prime}\right), 5.00$ $\left(\mathrm{d}, J=4.0 \mathrm{~Hz}, 0.3 \mathrm{H}, \mathrm{H}-1^{\prime \prime} \beta\right), 4.9(\mathrm{~d}, J=3.5 \mathrm{~Hz}, \mathrm{H}-3$ '), 4.1 (bs, 1H, H-4'), 3.89 (bs, 2H, H-5'), 3.24-3.68 (m, 6H, H-2", H-3", H-4", H-5"). ${ }^{31} \mathrm{P}$ NMR $\left(\mathrm{D}_{2} \mathrm{O}, 121.5 \mathrm{MHz}{ }^{1} \mathrm{H}-\right.$ decoupled) $\delta-8.86$. MS $\mathrm{m} / \mathrm{z}$ (-ve Electrospray) [100\% M$\mathrm{H}]^{-} 566,[49 \% \mathrm{M}-2 \mathrm{H}+\mathrm{Na}]^{-} 588.2\left(\mathrm{C}_{17} \mathrm{H}_{23} \mathrm{~N}_{5} \mathrm{O}_{16} \mathrm{P}_{2}\right.$ requires $M, 567$ ). MS $\mathrm{m} / z$ (+ve Electrospray) $[95 \% \mathrm{M}+\mathrm{H}]^{+} 568$, $[100 \% \mathrm{M}+\mathrm{Na}]^{+}, 590$. HRMS (LSIMS, positive) calcd for $\mathrm{C}_{17} \mathrm{H}_{22} \mathrm{O}_{13} \mathrm{~N}_{5} \mathrm{P}_{2}[\mathrm{M}+\mathrm{H}]^{-} 566.0689$ found 566.0681 .
Supplement: Acetylene $\beta-\left(2^{\prime}, 3^{\prime} O, O\right.$-isopropylidene)- 5'- $O$ (allyl ethynephosphonyl) D-adenosine

To a solution of $3(0.12 \mathrm{~g}, 0.42 \mathrm{mmol})$ and 2', 3'-O, $O-$ isopropylidene adenosine $(0.13 \mathrm{~g}, 0.42 \mathrm{mmol})$ in acetonitrile $(17 \mathrm{~mL})$ was added 2,4-DNP as a solid $(0.12 \mathrm{~g}$, $0.63 \mathrm{mmol})$. The reaction was stirred for $2 \mathrm{~h}$ after which time further quantities of $3(0.06 \mathrm{~g}, 0.21 \mathrm{mmol})$ and 2,4DNP $(0.04 \mathrm{~g}, 0.21 \mathrm{mmol})$ were added. The reaction was stirred for a further $3 \mathrm{~h}$ followed by further addition of the same amounts of $\mathbf{3}$ and 2,4-DNP. The reaction was almost completed after $10 \mathrm{~h}$ and the condensed intermediate was oxidized in situ by adding $t \mathrm{BuOOH}$ (solution 5-6 $\mathrm{M}$ in hexane, $0.084 \mathrm{~mL}$ ). After $7 \mathrm{~min}$, the reaction was quenched with a mixture of $\mathrm{CH}_{2} \mathrm{Cl}_{2}$ and a $\mathrm{NaHCO}_{3}$ saturated solution. The aqueous layer was extracted three times with $\mathrm{CH}_{2} \mathrm{Cl}_{2}$. The organic layers were combined, dried over $\mathrm{Na}_{2} \mathrm{SO}_{4}$ and concentrated under reduced pressure. The oily residue was chromatographed (silica, $\mathrm{CHCl}_{3} / \mathrm{MeOH}(92 / 8)$ ) to obtain a colorless oil (0.14 g, $0.28 \mathrm{mmol})$ which was directly dissolved in ethanol $(1.3 \mathrm{~mL})$. To the solution, $\mathrm{CsF}$ was added $(0.06 \mathrm{~g}, 0.39 \mathrm{mmol})$ and the reaction was stirred for $1 \mathrm{~h}$ at rt. A mixture DCM and saturated solution of $\mathrm{NaHCO}_{3}$ was then added and the aqueous layer was extracted three times with $\mathrm{CH}_{2} \mathrm{Cl}_{2}$. The combined organic layers were dried over $\mathrm{Na}_{2} \mathrm{SO}_{4}$ and concentrated under reduced pressure. The oily residue was chromatographed on silica to obtain the title compound as colourless oil in $57 \%$ yield $(0.103 \mathrm{~g}, 0.24 \mathrm{mmol}) .{ }^{1} \mathrm{H}$ NMR $\left(\mathrm{CDCl}_{3}, 300\right.$ MHz) $\delta 8.43(\mathrm{~d}, J=2.6 \mathrm{~Hz}, 1 \mathrm{H}, \mathrm{H}-2$ ade), 7.97 (d, $J=2.6$ $\mathrm{Hz}, 1 \mathrm{H}, \mathrm{H}-8$ ade), 6.21 (bs, $2 \mathrm{H}, \mathrm{NH}_{2}$-ad), 6.16 (d, $J=2.0$ Hz., $\left.1 \mathrm{H}, \mathrm{H}-1^{\prime}\right), 5.82-5.96\left(\mathrm{~m}, 1 \mathrm{H}, \mathrm{CH}_{2}=\mathrm{CHCH}_{2}\right), 5.42-5.46$ (m, $1 \mathrm{H}, \mathrm{H}-2$ ) $)$ 5.32-5.37 (m, $1 \mathrm{H}, \mathrm{CH}=\mathrm{CHCH}_{2}$ ), 5.23-5.26 $\left(\mathrm{m}, 1 \mathrm{H}, \mathrm{CH}=\mathrm{CHCH}_{2}\right), 5.12-5.14\left(\mathrm{~m}, 1 \mathrm{H}, \mathrm{H}-3^{\prime}\right), 4.53-4.57$ (m, $\left.2 \mathrm{H}, \mathrm{CH}_{2}=\mathrm{CHCH}_{2}\right), 4.50-4.52(\mathrm{~m}, 1 \mathrm{H}, \mathrm{H}-4)$, 4.31-4.37 (m, 1H, H-5'), 4.28-4.29 (m, 1H, H-5') 2.93 (dd, $J=13.5$ $\mathrm{Hz}, 18.6 \mathrm{~Hz}, 1 \mathrm{H}, \mathrm{H}$-acetylene), 1.63 (s, 3H, $\mathrm{CH}_{3^{-}}$ isopropylidene), 1.39 (s, $3 \mathrm{H}, \mathrm{CH}_{3}$-isopropylidene). ${ }^{13} \mathrm{C}$ NMR $\left(\mathrm{CDCl}_{3}, 125.75 \mathrm{MHz}\right) \delta 155.6$ (C-6 ade),153.1 (C-2 ade), 149.2 (C-4 ade), 139.6 (d, $J=3.4 \mathrm{~Hz}, \mathrm{C}-8$ ade), 131.6 (d, $\left.J=6.9 \mathrm{~Hz}, \mathrm{H}_{2} \mathrm{C}=C \mathrm{H}-\mathrm{CH}_{2} \mathrm{O}\right), 120.0$ (C-5 ade), 119.0 $\left(\mathrm{H}_{2} \mathrm{C}=\mathrm{CH}-\mathrm{CH}_{2} \mathrm{O}\right), 114.5$ ( $C$-isopropylidene), 90.9 (d, $\mathrm{J}=$ $9.0 \mathrm{~Hz}, \mathrm{C}-1$ ade), 89.2 (d, $J=52.1 \mathrm{~Hz}, \mathrm{C}-6$ acetylene), 85.2 ( d, $\left.J=8.6 \mathrm{~Hz}, \mathrm{C}-4^{\prime}\right), 85.1\left(\mathrm{~d}, J=8.7 \mathrm{~Hz}, \mathrm{C}-4^{\prime}\right), 84.2$ (C$\left.2^{\prime}\right), 81.4\left(\mathrm{~d}, J=4.9 \mathrm{~Hz}, \mathrm{C}-3^{\prime}\right), 74.4\left(\mathrm{~d},{ }^{3} J_{\mathrm{PC}}=9.7 \mathrm{~Hz}, \mathrm{C}-7\right.$ acetylene), $67.92\left(\mathrm{~d}, \mathrm{~J}=4.8 \mathrm{~Hz}, \mathrm{H}_{2} \mathrm{C}=\mathrm{CH}-\mathrm{CH}_{2}\right), 67.88$ (d, $\left.J=4.7 \mathrm{~Hz}, \mathrm{H}_{2} \mathrm{C}=\mathrm{CH}-\mathrm{CH}_{2}\right), 66.2$ (bs, C-5), $26.1\left(\mathrm{CH}_{3}-\right.$ isopropylidene $), \quad 24.3 \quad\left(\mathrm{CH}_{3}\right.$-isopropylidene). ${ }^{31} \mathrm{P} \quad \mathrm{NMR}$ $\left(\mathrm{CDCl}_{3}, 121.5 \mathrm{MHz}{ }^{1} \mathrm{H}\right.$-decoupled) $\delta-7.16,-7.33,-7.36$. HRMS (LSIMS, positive) calcd for $\mathrm{C}_{18} \mathrm{H}_{22} \mathrm{O}_{6} \mathrm{~N}_{5} \mathrm{P}[\mathrm{M}+\mathrm{H}]^{+}$ 436.1385 found 436.1399 . 